\title{
Milde Hypoglykämien erhöhen Mortalität nicht
}

Fragestellung: Wie häufig sind selbst berichtete Hypoglykämien (ohne Blutzuckermessung-Messung) bei Typ-2-Diabetikern mit Insulintherapie und sind sie mit der Mortalität assoziiert?

Hintergrund: Selbst berichtete Hypoglykämien sind nur selten Outcome-Parameter in Studien zu Typ-2-Diabetes und Insulintherapie. Üblicherweise werden nur bestätigte, d.h. gemessene Hypoglykämien für Studien herangezogen. Objektiv gemessene Hypoglykämien, vor allem schwere, sind in vielen Studien mit erhöhter Mortalität assoziiert [1, 2, 3]. Allerdings können nicht alle Patienten regelmäßig eine Glukoseselbstkontrolle machen, sodass selbst berichtete Hypoglykämien (ohne Messung) die Problematik im Alltag besser repräsentieren könnten.

Patienten u. Methoden: In dieser prospektiven Kohortenstudie wurden klinische Behandlungsdaten von 1646 Typ-2-Diabetikern mit Insulintherapie über einen medianen Follow-up von

Originalie

Rauh SP, Rutters F, Thorsted BL, et al. Self-reported hypoglycaemia in patients with type 2 diabetes treated with insulin in the Hoorn Diabetes Care System Cohort, the Netherlands: a prospective cohort study. BMJ Open. 2016;6(9):e012793.-22. 1,9 Jahren mit dem spezifischen Blick auf „berichtete“ Hypoglykämien ausgewertet.

Ergebnisse: Im Beobachtungszeitraum starben $98 \mathrm{~Pa}$ tienten (5,9\%). Milde Hypoglykämien waren hier mit einem signifikant niedrigeren Mortalitätsrisiko verbunden

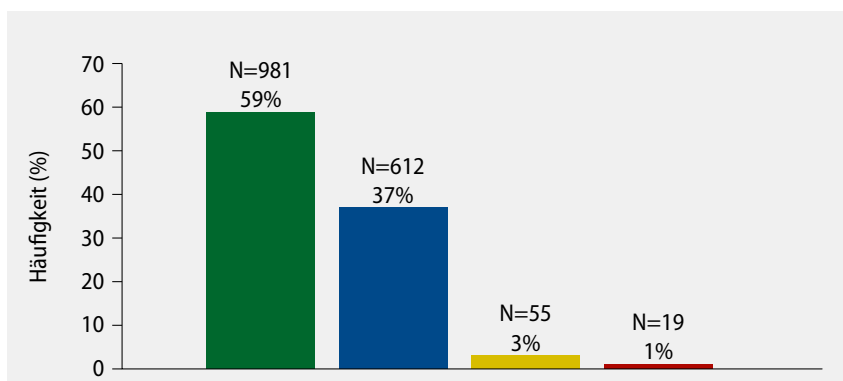

keine Hypoglykämie berichtet

Nur schwere Hypoglykämie berichtet Nur milde Hypoglykämie berichtet

Milde und schwere Hypoglykämie berichtet

1 Baselinedaten zu „gefühlten“ Hypoglykämien bei Patienten mit Typ-2-Diabetes und Insulintherapie.

(OD 0,48, 95\%-KI 0,28-0,80), schwere Hypoglykämien (Fremdhilfe) waren nicht mit der Mortalität assoziiert (OR 0,76, 95\%KI 0,33-1,80). Nach Adjustierung für demografische und klinische Merkmale ergab sich für schwere Hypoglykämien zwar eine erhöhte OR von 1,38 (95\%-KI 0,31-6,11) jedoch wurde das Signifikanzniveau verpasst.

Schlussfolgerung: Insulinbehandelte Typ-2-Diabetiker berichteten von vielen milden Hypoglykämien ( $\triangleright$ Abb. 1$)$, dies ist aber nicht mit erhöhter Mortalität assoziiert, eher im Gegenteil.

\section{- Kommentar von Dr. med. Thorsten Siegmund}

\section{Leider ein etwas kraftloses Ergebnis}

Trotz des interessanten Ansatzes, "gefühlte“ Hypoglykämien auf ihre Relevanz hinsichtlich des Sterberisikos zu untersuchen, weist die Studie doch einige Schwächen auf. Zunächst handelt es sich um eine reine Observationsstudie. Zweitens birgt die Definition „gefühlte Hypoglykämie" einen großen Unsicherheitsfaktor. Drittens lässt die an die kurze Studiendauer gekoppelte geringe Sterberate bei gleichzeitig geringer Zahl an schweren Hypoglykämien die notwendige Power zur Beurteilung einer Assoziation von schweren Hypoglykämien mit Mor-

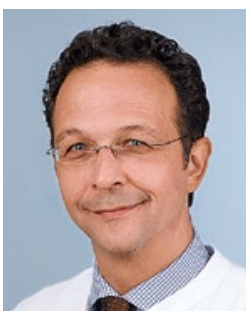

Dr. med. Thorsten Siegmund

Endokrinologie, Diabetologie und Stoffwechsel

Isar Klinikum München GmbH

Sonnenstr. 24-26, 80331 München thorsten.siegmund@isarklinikum.de talität vermissen. Beruhigend ist jedoch das Ergebnis, dass milde Hypoglykämien offensichtlich keine Assoziation zur Mortalität aufweisen. Valide Aussagen bzw. Berechnungen zur Assoziation von Hypoglykämien und Mortalität werden letztlich erst dann möglich sein, wenn Untersuchungen mit kontinuierlicher Glukosesensormessung vorliegen, der einzigen Methode, die jede Art und Schwere von Hypoglykämien lückenlos dokumentiert. Bis wir an solche Daten kommen, werden vermutlich jedoch noch viele Jahre vergehen.

\section{Literatur:}

1. Bonds DE, Miller ME, Bergenstal RM, et al. The association between symptomatic, severe hypoglycaemia and mortality in type 2 diabetes: retrospective epidemiological analysis of the ACCORD study. BMJ 2010;340:b4909.

2. Zoungas S, Patel A, Chalmers J, et al. Severe hypoglycemia and risks of vascular events and death. N Engl J Med 2010;363:1410-8.

3. Hsu PF, Sung SH, Cheng HM, et al. Association of clinical symptomatic hypoglycemia with cardiovascular events and total mortality in type 2 diabetes: a nationwide population-based study. Diabetes Care 2013:36:894-900. 